\title{
THE PROTOTYPE MESSAGE BROADCAST SYSTEM FOR THE SUPERCONDUCTING SUPER COLLIDER
}

\author{
Keng Low and Robert Skegg \\ Accelerator Division \\ Superconducting Super Collider Laboratory* \\ 2550 Beckleymeade Avenue \\ Dallas, Texas 75237
}

November 1990

\begin{abstract}
DISCLAIMER
This report was prepared as an account of work sponsored by an agency of the United States Government. Neither the United States Government nor any agency thereof, nor any of their employees, makes any warranty, express or implied, or assumes any legal liability or responsibility for the accuracy, completeness, or usefulness of any information, apparatus, product, or process disclosed, or represents that, its use would not infringe privately owned rights. Reference herein to any specific commercial product, process, or service by trade name, trademark, manufacturer, or otherwise does not neressarily constitute or imply its endorsement, recommendation, or favoring by the United States Government or any agency thereof. The views and opinions of authors expressed herein do not necessarily state or reflect those of the United States Government or any agency thereof.
\end{abstract}

* Operated by the Universities Research Association, Inc., for the U.S. Department of Energy under Contract No. DE-AC02-89ER40486. 


\title{
THE PROTOTYPE MESSAGE BROADCAST SYSTEM FO? THE SUPERCONDUCTING SUPER COLLIDER
}

\author{
Keng Low and Robert Skegg \\ Accelerator Division \\ Superconducting Super Collider Laboratory* \\ Dallas, Texas 75237
}

\begin{abstract}
A prototype unified message broadcast system to handle the site-wide distribution of all control system messages for the Superconducting Super Collider is presented. The messages are assembled in the control room area and encapsulated for transmission via a general fiber-optic link system to devices distributed throughout 70 miles of tunnels. An embedded timing signal is used by the distribution system to ensure that messages arrive at all devices simultaneously. Devices receive messages using a special receiver sub-system. A simple version of this system is to be used in the Accelerator Systems String Test (ASST) at the SSC site in 1991.
\end{abstract}

\footnotetext{
*Operated by the Universities Research Association, Inc., for the U.S. Department of Energy under Contract No. DE-AC02-89ER40486.
} 


\section{INTRODUCTION}

The Superconducting Super Collider (SSC) is a $20 \mathrm{TeV}+20 \mathrm{TeV}$ proton collider proposed for construction in the area of Waxahachie, Texas, during the next 10 years. The SSC consists of six accelerators: a linac, three booster synchrotrons and two intersecting, contra-rotating synchrotrons that make up the collider itself. The complex will occupy approximately 70 miles $(112 \mathrm{~km})$ of underground tunnels and is illustrated diagrammatically in Figire 1. There are estimated to be about 20,000 devices requiring remote control and interrogation in order to operate the accelerator and diagnose its condition. Tools that are being investigated to perform these tasks are time-division multiplex (TDM) transport of digital data by fiber-optic cable, reflective memory techniques for point-to-point data transmission, and a message broadcar' system (MBS) for distribution of medium-time-critical commands and data.

In large accelerator control systems, general data and commands have to be sent to many devices and a broadcast system is an efficierit way to do this. Such systems have been used successfully at FNAL [1] and CERN [2]. The MBS proposed for use at the SSC will carry only commands, brief data and rough timing. Voluminous operating data is to be transported by reflective memory or local area networks, and precision timing is to be handled by a dedicated system [3].

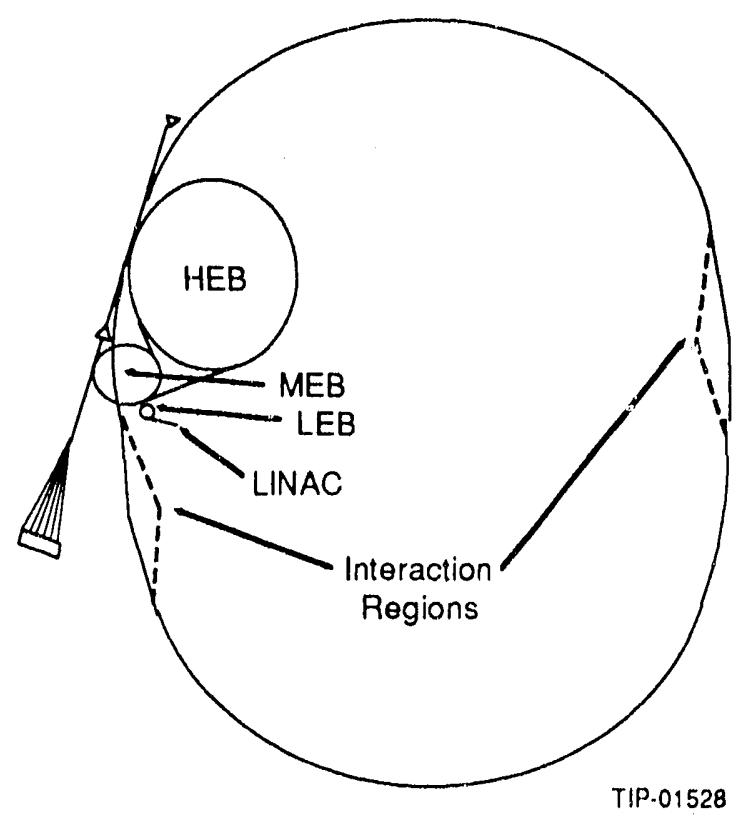

Figure i. The Superconducting Super Collider (not to scale)

\section{MESSAGE BROADCAST SYSTEM}

The MBS is shown in Figure 2 and consists of one central message generation system, a transport network, timing correctors and many receivers attached to controlled devices. The transport network contains no intelligent routing mechanisms; messages are simply broadcast to all receivers. Every broadcast message has a message type or identifier header together with some trailing bytes, the number of which is indicated in the message header. Each receiver is preprogrammed to recognize a set of one or more such messages. When a receiver identifies such a message it passes the complete message to the device for execution. 
Initial design plans call for separate broadcast systems for each of the six machines, since the machines have vastly differing cycle times, ranging from $0.1 \mathrm{sec}$. to many hours. Site-wide broadcast, when required, is achieved by commanding each message generator of all six broadcast systems to send the same message.

Messages are structured using 8-bit bytes. Each message consists of a two byte header, indicating the message type, followed by from zero to thirteen bytes of parameters. The messages are prefixed by a start byte and suffixed by an idle byte to enable transportation by a general time division multiplex system. The format of a typical message is shown in Table 1.

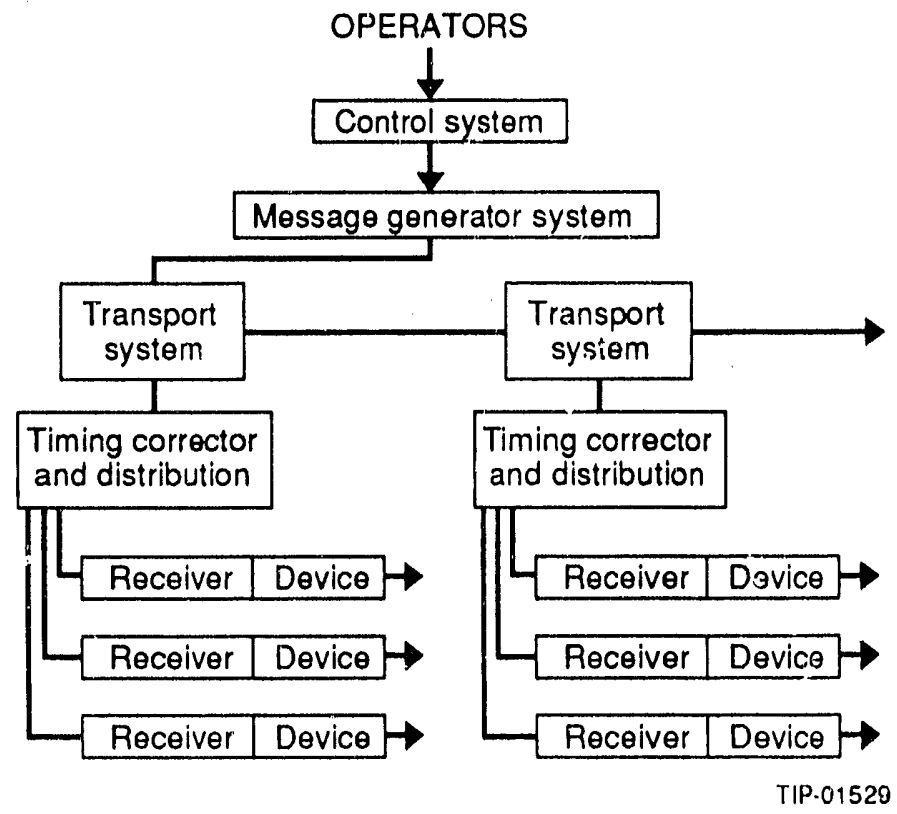

Figure 2. The Message Broadcast System.

Table 1. Message Format

\begin{tabular}{lcccccccc}
\hline & msb & & & & & & \multicolumn{2}{c}{ lsb } \\
\hline Start & 0 & 0 & 0 & 1 & 0 & 1 & 0 & 1 \\
Header & 0 & 1 & 1 & 0 & 0 & 0 & 1 & 1 \\
Header & 0 & 0 & 0 & 1 & 1 & 0 & 0 & 0 \\
Parameter & 0 & 1 & 0 & 0 & 1 & 0 & 0 & 1 \\
Parameter & 0 & 1 & 0 & 0 & 1 & 0 & 1 & 0 \\
Parameter & 0 & 1 & 0 & 0 & 1 & 0 & 1 & 1 \\
Idle & 1 & 1 & 0 & 0 & 1 & 1 & 0 & 0 \\
\hline \hline
\end{tabular}

The format of the start and idle bytes are shown in Table 2. The two most significant bits in the start byte are set to zero to assist in identifying the start of a message, the next four bits indicate the message length, and the final two bits are the byte check-sum. The message length includes the two byte header, allowing up to 13 parameter bytes, which is judged to be sufficient for all applications. The message length is thus the number of bytes before the terminating idle byte. The idle byte is binary 11001100 ; it follows every message and is also transmitted as fill when there are no messages. 
Table 2. Characteristics of Start and Idle Bytes

\begin{tabular}{lccccccccc}
\hline & $\mathrm{msb}$ & & & & & & \multicolumn{2}{c}{ lsb } \\
\hline Idle Byte & 1 & 1 & 0 & 0 & 1 & 1 & 0 & 0 \\
Start Byte & 0 & 0 & $\mathrm{n}$ & $\mathrm{n}$ & $\mathrm{n}$ & $\mathrm{n}$ & $\mathrm{c}$ & $\mathrm{c}$ \\
\hline \hline
\end{tabular}

nnnn = number of following bytes in message, $c c=$ checksum

This format allows for easy identification and integrity checking of messages. A message starts when an idle byte is followed by a byte containing ' 00 ' in the two most significant bits. This is the start byte, and can be checked for integrity using the checksum. The message length is extracted and decremented as the following header and parameter bytes are received. When the message length has been decremented to zero, an idle byte is expecied; otherwise a framing error has occurred. Data contained in the optional parameters may be checked for integrity by embedding check bits.

\section{MESSAGE GENERATION}

Messages are generated by a central system consisting of a single message sequence generator and six message formatters, one for each broadcast network. This system is shown in Figure 3. Sequences of messages to operate the various accelerator systems in tandem are predefined by physicists and are contained in a database. To achieve a required operational state, the master sequence generator uses this database to translate high level requests from operators to suitable sequences of low-level messages. These sequences are automatically transmitted by the message formatters to the accelerators.

Asynchronous requests and feedback control messages can also be handled by this system. Such requests might include luminosity measurements on demand, turn-by-turn chromaticity values for magnet ramp feedback control diagnosis, and 'beam dump' requests.

The sequence generator transfers the messages to the formatter using a common Randorn Access Memory (RAM). Each message formatter is assigned a separate block of this RAM, accessible only by itself and the message sequence generator. Messages are placed in RAM blocks assigned to the appropriate message formatter. The formatters read their blocks of RAM continuously, encapsulating and transmitting messages found there. Any asynchronous message sequences earmarked for a specific accelerator are transferred to special locations in RAM by the message sequence generator to be read by the appropriate formatter and transmitted over the broadcast network.

Every message table contains embedded instruction codes specifically for the message formatter. These instruction codes precede each message in the table. The message formatter recognizes these special instructions which it uses to control the transmission of messages.

The master sequence generator is a standard single computer board with a Motorola 68030 cpu. The message formatters each contain a special-purpose micro-controller which reads the common RAM, executes the embedded instructions and encapsulates messages for transmission.

An out of sequence message for immediate transmission may be placed by the master sequence generator in a special location in the common RAM. This message will be sent by the message formatter as soon as it finishes sending the current message. 
At a higher priority, special messages may be generated by a hardware signal. A transition on a line to the message formatter causes it to send a special pre-defined message from common RAM as soon as it reaches the end of its current message.

The message formatter also automatically produces a regular sync tick message. This is transmitted at a regular $1440 \mathrm{~Hz}$ rate $(694.44 \mu \mathrm{s}$ period) with an accuracy of a few microseconds, and is locked to the global precision timing system of the SSC [3]. It has two parameter bytes which contain the sync tick number. This message is used by the broadcast message distribution system for compensation of transmission delays, and may also be used as a mediumprecision timing signal by devices in the field. Devices requiring more accurate timing will use the global timing system of the SSC.

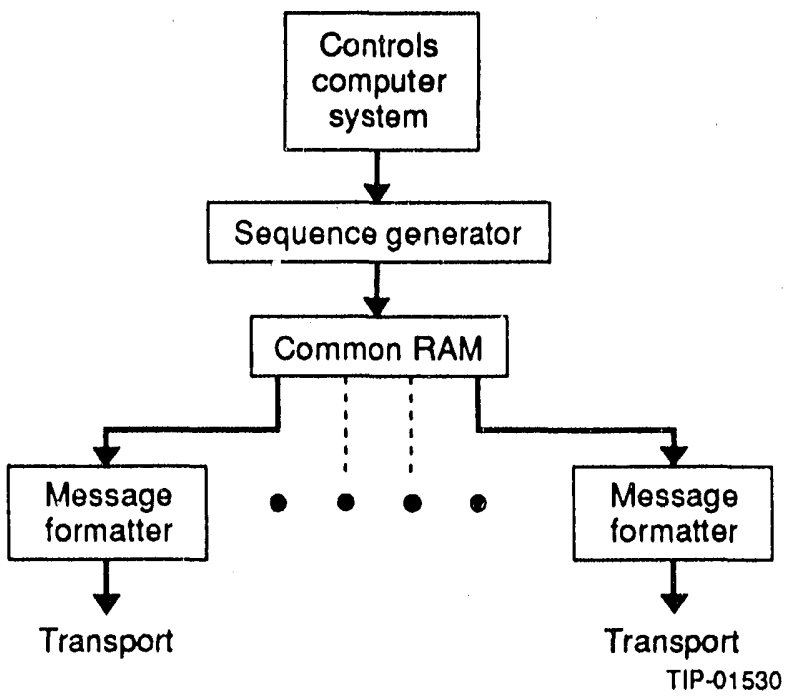

Figure 3. The Messsage Generation System.

The sync ticks must be transmitted at the correct interval; therefore, the message formatter must be aware of the time left before the next sync tick each time it has a message to send. If there is insufficient time remaining to send the next message before the next sync tick is due, then the message must be deferred until after the sync tick. A flow diagram of the tasks performed by the message formatter is shown in Figure 4.

\section{MESSAGE DISTRIBUTION AND RECEPTION}

\section{A. Message Transport}

The broadcast messages are designed to be transported by time-division multiplexing on fiber optic links, as used by the telecommunications industry. It is expected that one $\mathrm{T} 1(1.544 \mathrm{Mb} / \mathrm{s})$ channel will be used, providing a capacity of about 26,000 messages-per-second, where an average message consists of a start byte, two header bytes, three parameter bytes and an idle byte. 


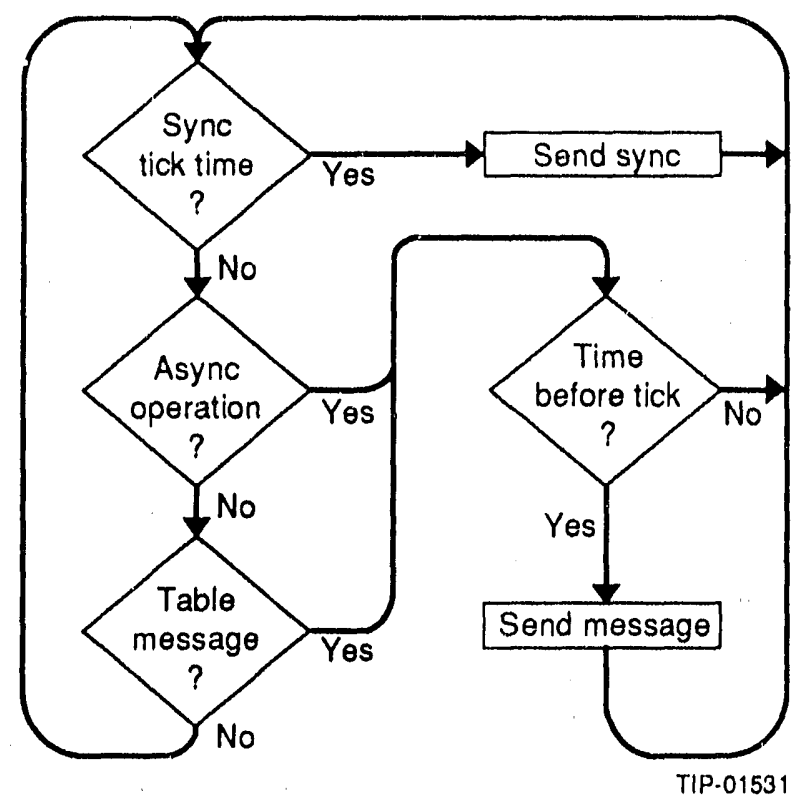

Figure 4. Flow Diagram for the Message Formatter

Due to the large area covered by the SSC and delays caused by the many repeaters in the transport system, a time-skew of several milli-seconds will occur in the arrival of messages at local and remote points. To ensure that broadcast messages arrive at all devices simultaneously, a timing corrector is located at each message broadcast system distribution point. (See Figure 2).

The timing corrector compares timing signals (sync ticks) embedded in the broadcast message stream with the local precision timing system signals and inserts a correcting delay so that any broadcast message will arrive at all locations with a skew of only a few micro-seconds. Messages are prepared and transmitted from the control room ahead of the required execution time to allow for this delay. Actions which require more accurate timing than the message broadcast system can provide will use trigger signals from the global precision timing system.

\section{B. Broadcast Message Receivers}

Devices which are to receive messages via the message broadcast system do so using a Broadcast Message Receiver (BMR.) This sub-system is connected to the transportation system and monitors all broadcast messages. It recognizes those messages which are of interest to the device, stores the message header and any associated parameters, and sends a signal to the device indicating that a message has been received. The device then interprets the message header and associated parameters, and performs the required action.

The BMR may be used as a micro-processor peripheral or as a simple stand-alone interface. As an intelligent peripheral it connects to the address and data bus, sharing access to RAM (See Figure 5). A table of the types of messages that the device wishes to receive is placed in the RAM by the micro-processor and accessed by the BMR. Parameters associated with received messages are placed in RAM by the BMR for use by the micro-processor. The BMR produces an interrupt to the micro-processor when a message of interest has been received. Several messages may be buffered in the RAM for eventual processing. 


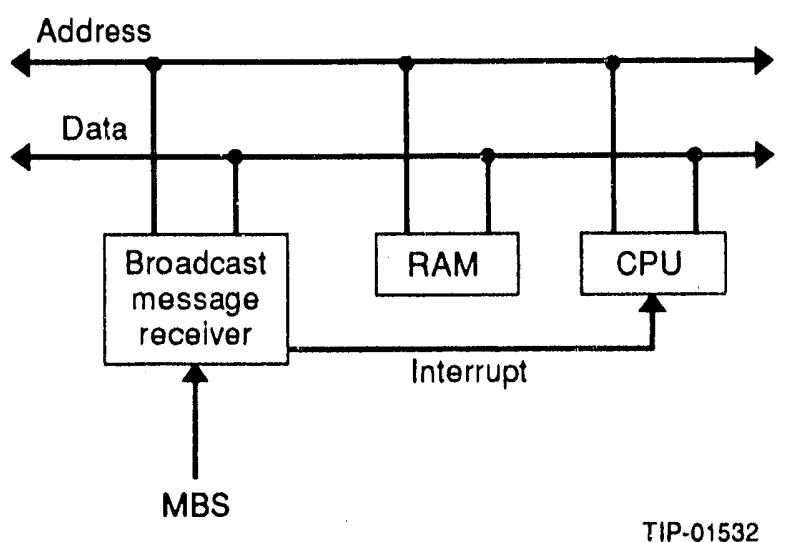

Figure 5. Broadcast Message Receiver With Micro-Processur

In simple applications the BMR may be used without a micro-processor. In this case either a switch or jumper system may be used to indicate one or two valid message types, or a PROM may be used for more complex recognition. Parameters may be stored in external byte-wide Dlatches. (See Figure 6).

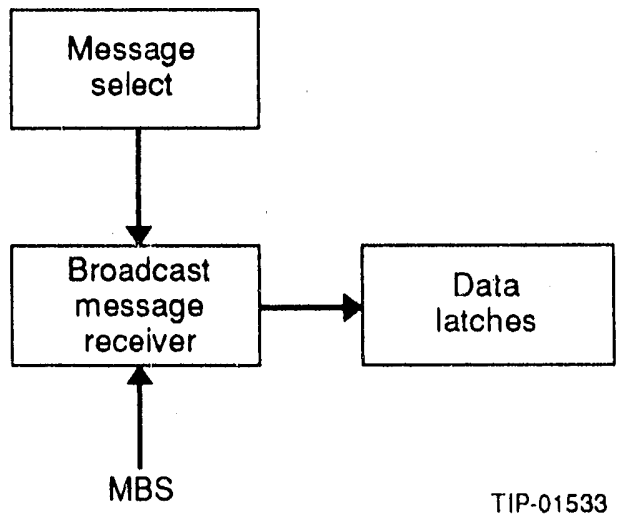

Figure 6. Simple Broádcast Message Receiver

\section{CURRENT STATUS}

Detailed design work is under way for a simple version of the message broadcast system to be used in the Accelerator Systems String Test at the SSC site in 1991. The ASST is a feasibility study of a set of cryogenically cooled superconducting magnets. The messages will be of fixedformat type, containing two parameter bytes. Receivers will recognize one programmable message type, and output eight binary control signals. The system will comprise one message generator and several message receivers, interconnected by a shielded twisted-pair cable, with transformer isolation. Signalling will be T1 format at $1.544 \mathrm{Mb} / \mathrm{s}$.

The proposed hardware configuration will consist of a Mizar 8130 (MZ8130) single board computer with a $68030 \mathrm{cpu}$ functioning as the central message generator and a custom microcontroller for the message formatter.

VxWorks has been chosen as the software development environment since it provides for a real time, high performance operating system for the MZ8130. As a cross-development system, VxWorks also provides a rich software development environment under Unix. 


\section{ACKNOWLEDGMENTS}

We thank H. Lue and C. Saltmarsh for their contributions to the design and development of this system.

\section{REFERENCES}

1. R. J. Ducar et al., "FNAL Booster Intensity, Extraction, and Synchronization Control For Collider Operation", 1987 Particle Accelerator Conference, Washington D.C.

2. C. G. Beetham et al., "Overview of the SPS/LEP Fast Broadcast Message Timing System", 1987 Particle Accelerator Conference, Washington D.C.

3. D. J. Martin et al., Early Instrumentacion Projects at the SSC, SSC Laboratory Report SSCL-272 (April 1990). 

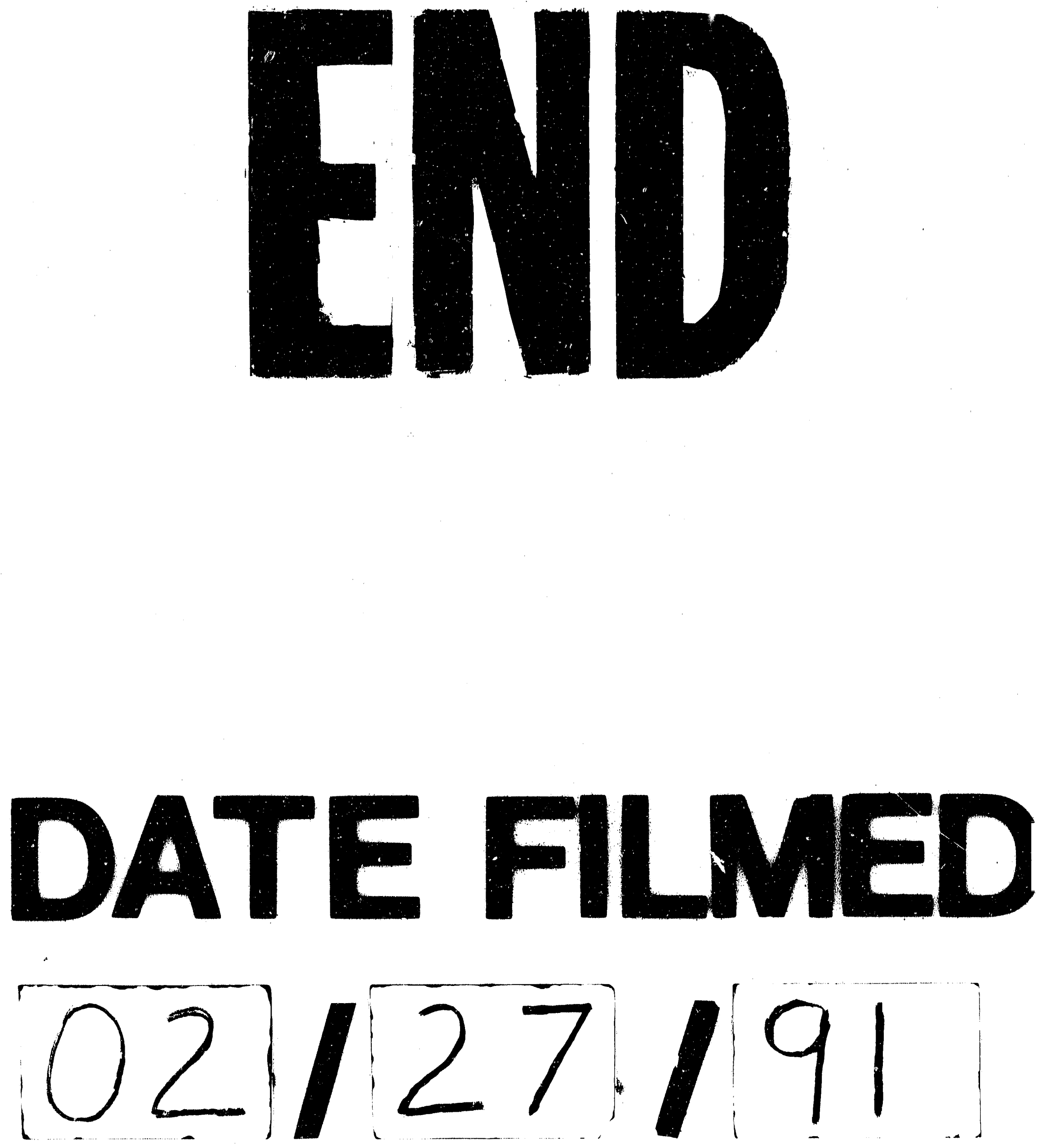
$-$ 